\title{
Study on the Broadcast Beam-forming of Smart Antenna by the Method of Baseband-Amplitude-Weighting
}

\author{
Haiyang Fu, Honggang He, Xiangdong Jia \\ College of Communication and Information Engineering, \\ Nanjing University of Posts and Telecommunications, \\ Nanjing, China \\ cholemoretz@gmail.com
}

\begin{abstract}
In reality, wireless communication channels are designed to serve for multiple users while multipath propagation interference (MPI) and other problems are deteriorating the quality of service, especially when modern wireless communication demands higher data rate giving that less and less radio spectrum resources are available. Compared to conventional techniques trying to solve the related problems, smart antenna (SA) may have shed new light on the solutions of these issues. The forming of broadcast beam of SA is studied in this paper. Firstly, the mathematical formulas for the forming of directional broadcast beam by the method of baseband-amplitude-weighting are derived, according to which the corresponding circuit diagram is given. Then the forming method for broadcast beam for SA by combining several directional radio beams is presented. Moreover, the computer simulation results of SA with 6 array elements are provided to demonstrate the accuracy and achievability of this beamforming method.
\end{abstract}

Keywords- Broadcast beam-forming; QPSK; smart antenna; baseband-amplitude-weighting

\section{INTRODUCTION}

In wireless communications, frequency efficiencies and power utilization are among the most important concerns of system designers. Smart antenna (SA), with the function to form directional transmission of signals, becomes an effective method to enhance spectrum efficiency as well as power utilization of wireless communication systems. With SA, spatial division multiple access (SDMA) can be realized, which can be a help to reduce the bad effects of multi-path interference (MPI) as well as multiple access interference (MAI), thus to offer a better system quality. The broadcast beam-forming is used for cell coverage. The published schemes for broadcast beam-forming of SA include the scanning method and the synthesis method. The so-called scanning method in [1] divides a cell along the horizontal direction evenly into $\mathrm{N}$ (a certain integer) aliquots, each aliquot as a sub-cell; each sub-cell, respectively, is covered by a narrow beam; then starting with one of these sub-cells, make the narrow beam of the sub-cell last for the time equal to the maximum period of common information, during which the same narrow beam-forming operation is applied to both common information and business information. After the end of the dwelling time, narrow beam-forming is switched to the next sub-cell; so turn the cycle turns, the entire cell can get covered by broadcast beams. References $[2,3]$ proposed a dynamic sector broadcast beam-forming realization method which can be referred to as the synthesis method and gave its corresponding apparatus. This method demands to be informed of the spatial distribution characteristics of the traffic load in order to determine the pointing direction of the broadcast beam of the dynamic sectors and the range of angular coverage, on the basis of which the directions of the beam-forming for a plurality of conventional business are defined with a certain angle interval, followed by other further processing steps to achieve the dynamic broadcast beam for a sector.

In the scanning method, the mobility of the mobile terminals is not fully taken into consideration; In the case of vehicle wireless communication system, a mismatch would be caused between the narrow beam and a terminal moving in a high speed between sub-cells, that is, the narrow broadcast beam is "catching up" with the mobile terminal while the broadcasted information cannot really be detected by it. The feature of the synthesis method lies in the need to obtain the spatial distribution characteristics of the traffic load; while the broadcast channel should be ties of the mobile terminals and base station to establish the initial contact, to form the broadcast beam using the spatial distribution characteristics of business traffic is like to put a cart before the horse.

It seems, from above discussions, that some methods for broadcast beam-forming have, more or less, defects. Given that circumstance, this paper tries to present a broadcast beam-forming of QPSK modulation by basebandamplitude-weighting, which can be used in TD-SCDMA. This method proposed by us is capable of satisfying the requirements of broadcast beam-forming, because of which spectral efficiency and transmitting power utilization can be improved. And moreover, we know that there are generally three ways to realize directional beam-forming in reality which are radio frequency (RF) method, intermediate frequency (IF) method and baseband method. However, to complete phase shifting in precision when frequency is very high is quite difficult; since the method to form broadcast beam put forward by us also can be used for the directional beam of code channels, the beam-forming for SA has been made easier to implement, making systems utilizing our method have an increase of equipment reliability, a decrease of fees and the popularization of SA much more possible, 
compared to RF or IF realization methods such as in $[9,10$, 11] and other published patents in $[1,2,3,4]$.

\section{PRINCIPLE AND IMPLEMENTATION SCHEME OF BEAM- FORMING BY BASEBAND-AMPLITUDE-WEIGHTING}

It is according to the principle of phase controlled array (PCA) that the SA directional transmission is realized, and based on the same principle, the current phase of the nth antenna array element (AE) of a linear array of SA is as follows:

$$
\theta_{\mathrm{n}}=(\mathrm{n}-1) \pi \sin \phi_{0},
$$

in which $\Phi_{0}$ denotes the direction in which the main lobe of the transmitted beam gets its maximum value. It can be told from (1) that the directional transmission of signals is possible to be achieved by inserting an opposite phase to the nth $\mathrm{AE}$ to offset the current phase, however, as we have mentioned in section $I$ that it is very difficult to realize complete and accurate phase shift for the RF and IF; therefore, baseband processing might be a good choice to realize directional (broadcast) beam-forming.

From the principle of QPSK modulation, the expressions of the In-phase and Quadrature-phase carrier, respectively, are as follows:

$$
\mathrm{I}: \cos (\omega \mathrm{t}-\theta), \quad \mathrm{Q}: \sin (\omega \mathrm{t}-\theta) .
$$

Thus their complex forms are,

$$
\mathrm{I}: \operatorname{Re}\left[e^{j \omega t} e^{-j \theta}\right], \quad \mathrm{Q}: \operatorname{Im}\left[e^{j \omega t} e^{-j \theta}\right],
$$

in which $\mathrm{Re}[$.$] represents for the real part of a complex$ number and $\operatorname{Im}[$.$] for the imaginary part.$

With (2), (3) and the use of both the principle of quadrature modulation and multi-beam superstition theorem, the mathematical expression of directional transmitting beam-forming for a linear array of SA by the method of baseband-signal-weighting can be deduced and the result is as follows:

$$
\operatorname{Re}\left[e^{-j \theta}\right] \cos \omega t-\operatorname{Im}\left[e^{-j \theta}\right] \sin \omega t
$$

in which $\theta$ denotes the current phase of the specific AE, and $\omega$ is the carrier angular frequency.

With the assistance of (4), it is possible to design the device for unidirectional transmission of signal beams for a single code division multiple access (CDMA) code channel using our beam-forming method. The ideal broadcast beamforming for a linear SA array can be acquired by the combining of directional transmitted beams in 8 different directions within one code channel, where the eight directions(denoted by $\phi_{0}$ in (1) ) are taken as, respectively, $47^{\circ},-47^{\circ}, 33^{\circ},-33^{\circ}, 20^{\circ},-20^{\circ}, 7^{\circ}$ and $-7^{\circ}$. The core part of the apparatus for broadcast beam by the method of QPSK baseband signal weighting with one AE of a linear SA array is shown in Fig.1 which includes the serial-parallel converting circuit 11; weighting-coefficients-generating circuit 12 which determines the weighting coefficients of the signal in the transmitting direction; multipliers $M_{13}, M_{14}, M_{15}$ and $M_{16}$ for the multiplication of baseband data and the real or imaginary parts of the weighting coefficients from circuit 12 to form the signals $I_{I 11}, Q_{I 11}, Q_{Q 11}$, and $I_{Q 11}$; multipliers $M_{19}$ and $M_{20}$ for quadrature modulation of weighted baseband signals; an adder $M_{21}$ for producing quadrature modulated signals;90-degree-phase-shifting circuit 13 to shift the phase of carrier $\cos (\omega t)$; up-conversion circuit 14 to move the modulated baseband signal i.e. $Q P S K_{11}$, to a high frequency in order to be transmitted; and $A_{11}$, an $\mathrm{AE}$ of a linear SA array.

Moreover, based on (2) and (3), the following formulas can be obtained:

$$
\begin{aligned}
& \cos (\omega t-\theta)=\operatorname{Re}\left[e^{j \omega t} e^{-j \theta}\right] \\
= & \operatorname{Re}[(\cos \omega t+j \sin \omega t)(\cos \theta-j \sin \theta)] \\
= & \cos \theta \cos \omega t+\sin \theta \sin \omega t \\
= & \operatorname{Re}\left[e^{-j \theta}\right] \cos \omega t-\operatorname{Im}\left[e^{-j \theta}\right] \sin \omega t \\
& \sin (\omega t-\theta)=\operatorname{Im}\left[e^{j \omega t} e^{-j \theta}\right] \\
= & \operatorname{Im}[(\cos \omega t+j \sin \omega t)(\cos \theta+j \sin \theta)] \\
= & -\sin \theta \cos \omega t+\cos \theta \sin \omega t \\
= & \operatorname{Im}\left[e^{-j \theta}\right] \cos \omega t+R e\left[e^{-j \theta}\right] \sin \omega t
\end{aligned}
$$

Equations (5) and (6) can be utilized to give the schematic diagram of QPSK baseband weighting circuit for directional transmission with a linear array of SA.

Fig. 2 shows the main circuit structure of applying baseband-signal-weighting to generate directional transmission of signal beams for one AE of SA, which consists of the serialparallel converting circuit to output $I_{m}(t)$ and $Q_{m}(t)$; weighting coefficients generating circuit which determines the weighting coefficients of the signal in the transmitting direction; multipliers such as $M_{n 3}$ and $M_{n 4}$ for completing QPSK modulation while the adder $M_{n 11}$ is used to achieve the final baseband modulated signal $Q P S K_{11}$ which is then up-converted to a higher frequency to be transmitted through an AE, i.e. $A_{n}$. 

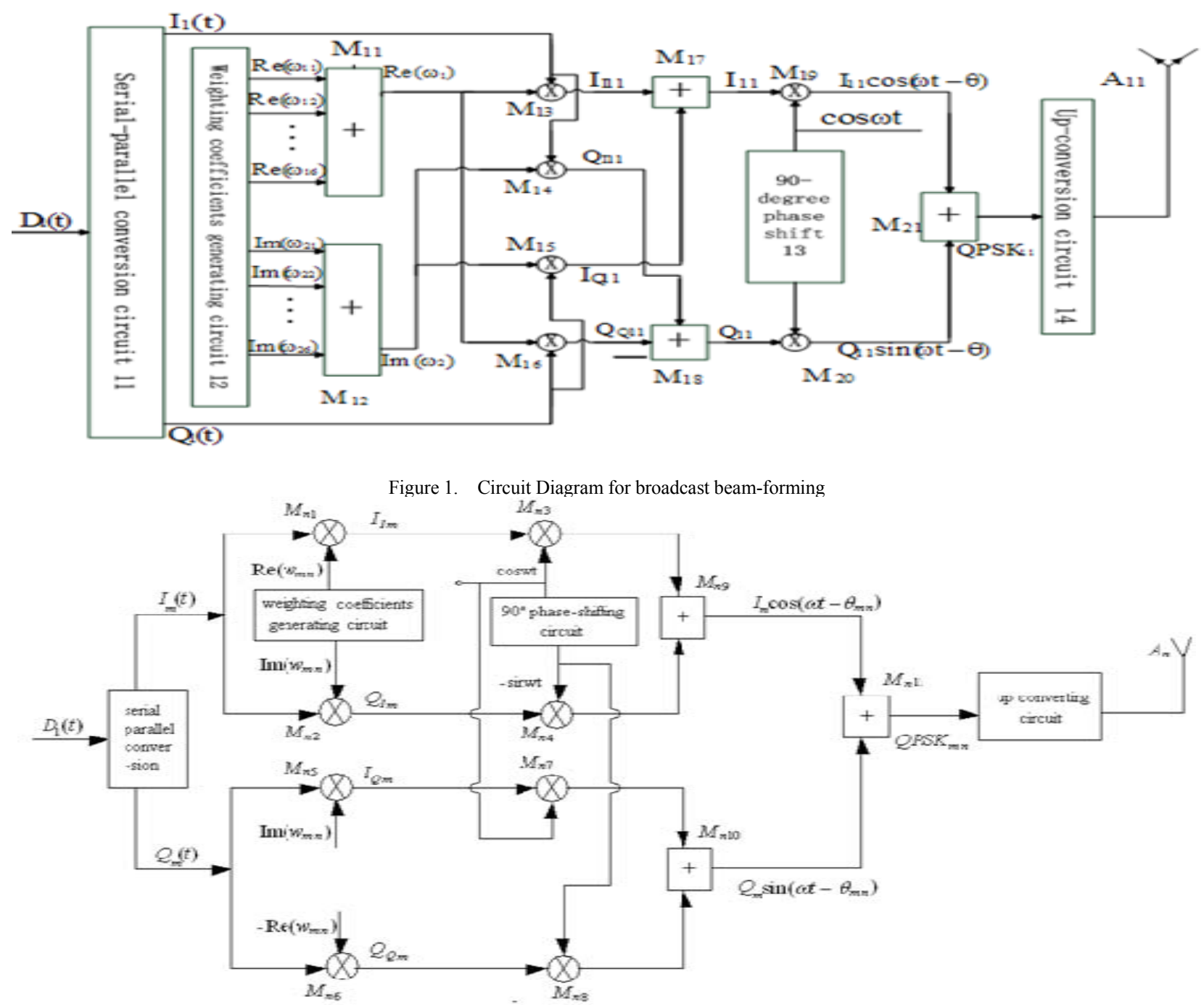

Figure 2. Single direction beam-forming circuit

In Fig.2, the $w_{m n}$ which is equal to $e^{-j \theta}$ in (5) and (6) means the coefficient of base-band-amplitude-Weighting. Another thing that must be pointed out here is $\mathrm{I}_{\mathrm{Im}}$ and $\mathrm{I}_{\mathrm{Qm}}$ together represent for the input data of path I in QPSK modulators, $m=1,2, \ldots, 6$ is the sign of the number of the code channels; Q $\mathrm{Im}$ and $\mathrm{Q}_{\mathrm{Qm}}$ have similar meanings. When there are 6 AEs in the linear SA array, 6 sets of similar circuit are needed.

With further considerations, a simplified version of Fig.2 can be achieved as shown in Fig.3. In Fig. 3, as we have mentioned before, $\mathrm{I}_{\mathrm{Im}}$ and $\mathrm{I}_{\mathrm{Qm}}$ are the input signals of QPSK modulator corresponding to the I path modulation; QIm and $\mathrm{Q}_{\mathrm{Qm}}$ are the input signals of QPSK modulator corresponding to the $\mathrm{Q}$ path modulation. By comparison of Fig.2 and Fig.3, it can be discovered a group of multipliers are eliminated using the principle of superposition theorem. As it can be predicted that with the simplified circuit, the device cost and complexity will be reduced when this apparatus is utilized to form broadcast beam in practice.

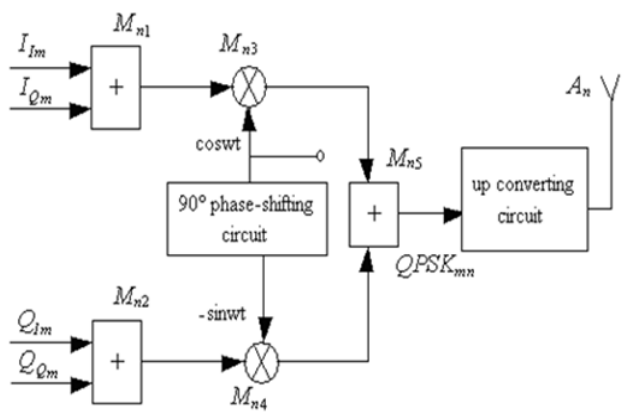

Figure 3. Simplified circuit blocks of beam-forming for single direction

\section{ANALYSIS AND SimUlation OF SySTEM PERFORMANCE}

To complete directional transmission, a linear SA array generally consists of six AEs, which means $I_{m}(t)$ and $Q_{m}(t)$ as illustrated in Fig.2, i.e. the outputs of the serial-parallel conversion circuit, need to be sent to 5 other signal processing units similar to the one aforementioned 
simultaneously. In these five processing units, the weightingcoefficients-generating circuits should generate the real parts of the weighting coefficients, $\operatorname{Re}\left(w_{2}\right), \operatorname{Re}\left(w_{3}\right), \ldots, \operatorname{Re}\left(w_{6}\right)$, and the imaginary parts, $\operatorname{Im}\left(w_{2}\right), \operatorname{Im}\left(w_{3}\right), \ldots, \operatorname{Im}\left(w_{6}\right)$, which, respectively, corresponds to those antenna elements $A_{2}$, $A_{3}, \ldots A_{6}$.

Fig. 4(a) shows the simulation result of a relatively ideal broadcast beam of a linear array of SA produced by the combining of 8 directionally transmitted beams, where the 8 directions are taken as, respectively, $47^{\circ}$ 、 $-47^{\circ}$ 、 $33^{\circ} 、-33^{\circ} 、 20^{\circ} 、-20^{\circ} 、 7^{\circ}$ and $-7^{\circ}$, which were denoted by $\phi_{0}$ in (1). It could be seen from Fig.4(a) that in the sector from $-60^{\circ}$ to $60^{\circ}$, which is required to be covered by broadcast beam, the amplitudes of the signal have been transmitted directionally and evenly. And it can be enhanced to obtain a better performance. When an antenna array with baffle-board is used, the covering area in the opposite direction of the aforementioned sector will be removed.

To give a better support for the broadcast beam-forming of SA by the baseband signal weighting method, another similar simulation result when 16 directional transmitted beams in 16 different directions which are combined to form an relatively ideal broadcast beam to cover a cell is shown in Fig.4(b).

It could be concluded from Fig.4(a) and Fig.4(b) that the method, to form broadcast beam with a linear array of SA, is effective to satisfy cell coverage in wireless communication systems.

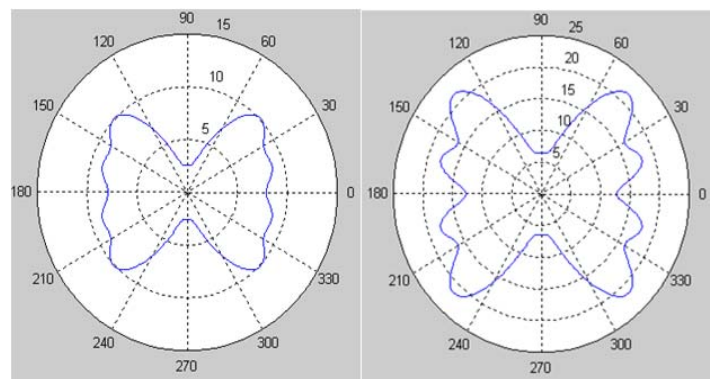

(a) 8-direction

(b) 16-direction

Figure 4 Simulation result of broadcast beam-forming by the combination of multi-direction beams

\section{SUMMARY}

In this paper, according to the expression of the current phase of the nth AE of a linear array of SA and QPSK modulation principles, the mathematical formulas for the forming of directional broadcast beam by the method of baseband-amplitude-weighting are derived, and the corresponding implementation scheme is given to make the broadcast beam-forming by the combination of several directional radio beams. Finally, the computer simulation results are presented which can serve as the evidence to demonstrate the accuracy and achievability of this beamforming method.

The circuit diagram for the CDMA broadcast beamforming by the method of baseband-amplitude-weighting is described in detail in the above sections, the use of each part of which, is based on solid theoretical foundations and can be easily understood. Moreover, computer simulation results demonstrate our method is an efficient one to achieve relatively ideal broadcast beam with effective cell coverage.

It can be seen from this paper the method to form broadcast beam with a linear array of SA for the CDMA broadcast channel by the method of QPSK baseband-signalweighting is practical and reliable. Compared with conventional broadcast beam-forming methods, our method is easier to be implemented, and will increase the reliability of related equipment with lower fees, making it possible to popularize SA.

\section{ACKNOWLEDGMENT}

This work was supported by the Research Fund for the Doctoral Program of Higher Education of China under Grant 20113223110001.

\section{REFERENCES}

[1] ZTE Corporation, A method of broadcast beam-forming in Smart Antenna, China, Patent application number: 200610031065.8(made public on Mar. 19th, 2008).

[2] ZTE Corporation, A method of dynamic sector broadcast beamforming, China, Patent application number: 200610137732.0(made public on Apr. 30th, 2008).

[3] ZTE Corporation, The apparatus of dynamic sector broadcast beamforming, China, Patent application number: 200610137729.9(made public on Apr. 30th, 2008).

[4] Datang Mobile Communications Equipment Co., Ltd., A method of broadcast beam-forming in systems of multi-antenna array and its apparatus, China, Patent application number: 200710119712.5(made public on Feb. 4th, 2009)

[5] JinYonghong, GengJunping, FanYu, Smart Antenna in wireless communications, Beijing: Press of Beijing University of Posts and Telecommunications, 2006.

[6] LiuMing, Technology and applications of Smart Antenna, Beijing: China Machinery Press, 2007.

[7] FanChangxin, Principle of communication,6th ed., Beijing: National Defense Industry Press, 2009.

[8] Yangwei, ChenShijun, LiShiming, JiangLianshan, The array antenna technology in mobile communication, Beijing: Beijing Jiaotong University Press, 2005.

[9] KangFengqi, "Study of wide beamforming in the Smart Antenna system," Electronic Technology, 2006, 9, pp.38-44.

[10] LuZhiyong, LiShuxue. "A design method of wide beam-forming antenna," Antenna Technology, 2003, Vol.29(6), pp.28-29.

[11] Lu Fenghui, Liu Yang, Li Xiaoming, "Performance analysis of system simulation on one kind of compact TD-SCDMA Smart Antenna," Telecommunication Engineering Technology and Standardization, pp.12-15, 2008.10. 